\title{
Bullying victimisation and psychosis: the interdependence and independence of risk trajectories
}

\author{
Gennaro Catone, Steven Marwaha, Belinda Lennox \& Matthew R. Broome
}

\begin{abstract}
SUMMARY
A number of studies have noted an association between being the victim of bullying and psychotic symptoms. We offer an overview of the topic, focusing especially on a developmental perspective. We highlight the results of the latest studies on psychosis across the continuum and its relationship with bullying. Then we summarise the three main explanatory models investigated: developmental, biological and cognitive. We recommend that bullying in psychosis requires careful study of the developmental trajectories involved, and that research should now focus on how personal, social and biological factors interact.
\end{abstract}

\section{LEARNING OBJECTIVES}

- Consider a spectrum of psychosis rather than a dichotomous concept of the disease (present/ absent)

- Understand that, by preventing bullying, it may also be possible to prevent the development of psychotic symptoms

- Reflect that psychotherapy helps to integrate past stressful experiences with current symptoms, providing an alternative interpretation of them

\section{DECLARATION OF INTEREST}

None

In recent years, research in psychosis has turned attention to social and environmental factors. In this context, a number of studies note an association between adversity, particularly stressful events, and psychosis (Reeder 2017). Varese et al (2012), in a meta-analysis, examined the association between childhood trauma (sexual, physical and emotional/ psychological abuse; neglect; parental death; and being bullied) and psychosis (identified by both diagnostic and dimensional measures), and concluded that there is a significant association $(\mathrm{OR}=2.78 ; 95 \%$ CI 2.34-3.31). Furthermore, Trotta et al (2015) showed in a review and meta-analysis that childhood adversities were associated also with persistence of psychotic symptoms.

Bullying is a specific stressful event, typically occurring in childhood and adolescence (Box 1). It has been defined as 'intentional aggressive behaviour that is repeated against a victim who cannot readily defend him- or herself' (Olweus 1993).

The relationship between bullying and psychosis has attracted much attention from researchers in an attempt to further understanding of the mechanisms underlying psychosis. In their meta-analysis, van Dam et al (2012) suggest that being bullied in childhood ('bullying victimisation') increases the risk of psychotic symptoms twofold, in a dose-response fashion; bullying is related to more persistent symptoms and the risk is further increased when victims of bullying are also perpetrators. Cunningham et al (2016) replicated this result in a meta-analysis that included prospective design studies on the relationship between bullying and psychosis.

The main aim of this article is to summarise the findings of studies on the link between bullying and psychosis as a continuum (Box 2). The psychosis continuum includes people with full-blown psychosis, those at ultra-high risk for the disorder (Box 3) and those in the general population with psychotic symptoms that do not reach the threshold for clinical impairment. Estimates suggest that the risk of transition to psychosis in ultra-high risk individuals ranges from $18 \%$ at 6 months to up to $32 \%$ by 3 years (Fusar-Poli 2012). The second aim is to frame the relationship between bullying and psychosis in a developmental perspective, exploring the main theoretical reasons for their connection.

\section{BOX 1 'Bullying'}

- Bullying can take different forms, such as direct (physical and verbal) and indirect (relational and social)

- Being a victim of bullying is associated with symptoms of mental illness. The risk is greater when the bullying occurs in childhood and adolescence, and it increases with the frequency, severity and duration of the bullying

- Peer victimisation can lead to acute distress, chronic worrying, nightmares and decreased well-being. It can also have long-term effects on mental health such as depression, anxiety, lower self-esteem, and suicide attempts and ideation
Gennaro Catone is a child and adolescent psychiatrist in the Department of Mental and Physical Health and Preventive Medicine, Università della Campania Luigi Vanvitelli, and a teaching assistant in the Faculty of Educational Sciences, Suor Orsola Benincasa University, Naples, Italy. He is interested in how biological and social factors interact during development and determine the onset of psychosis, and in the content and meaning of psychotic symptoms. Steven Marwaha is a Reader in Psychiatry at the Unit of Mental Health and Wellbeing, Warwick Medical School, and a consultant psychiatrist in Coventry and Warwickshire Partnership Trust. His interests include early intervention in bipolar disorder and psychosis, and mood dysregulation. Belinda Lennox is Associate Professor in the Department of Psychiatry, University of Oxford, and an honorary consultant psychiatrist in the Oxford early intervention in psychosis service. Her research interests are in exploring the causes of early psychosis and developing new treatments for it. Matthew Broome is Chair in Psychiatry and Youth Mental Health and Director of the Institute for Mental Health in the University of Birmingham's School of Psychology. He is also a member of the Department of Psychiatry and Faculty of Philosophy at the University of Oxford. His research interests include the prodromal phase of psychosis, delusion formation, functional neuroimaging, the role of GABA in psychotic symptoms, mood instability, and the philosophy of psychiatry. Correspondence Dr Gennaro Catone, Department of Mental and Physical Health and Preventive Medicine, Università della Campania "Luigi Vanvitelli", Via Pansini 5, Napoli NA 80100, Italy. Email: catoge@libero.it

\section{Copyright and usage}

(C) The Royal College of Psychiatrists 2017. 
BOX 2 The psychosis continuum

Research into psychosis has incorporated the concept of a spectrum or continuum of psychotic illness. Psychosis covers a range of experiences, and this approach posits that these lie along a continuum, with psychotic-like experiences at one end and full-blown psychosis and its related syndromes/disorders (e.g. schizophrenia, affective psychosis) at the other.

\section{The relationship between bullying and the psychosis continuum}

\section{Bullying and psychotic illness}

Psychotic illness and schizophrenia have long been topics of study in social psychiatry. In recent years, research has once again embraced wider factors in the mechanisms of psychosis and has moved to a biopsychosocial model that expands the purely biological explanatory model by drawing on cognitive psychology and social psychiatry (Broome 2005). Bebbington et al (2004), analysing the second British Adult Psychiatric Morbidity Survey (APMS), found that people with psychosis showed a prevalence of bullying victimisation four times higher than respondents without psychotic disorder, but this effect disappeared after adjustment for other traumatic life events. These findings have been more recently replicated in analyses of the second and also the third APMS (Catone 2015). A review by van Dam et al (2012) highlights another longitudinal study with a baseline for assessing bullying victimisation at 8 years of age and followup after 10-15 years found that 13 of the 2540 participants had developed a psychotic disorder and $2.9 \%$ of these had the status of bullying victim/ perpetrator. The effect disappeared when parental education level and participants' general emotional and behavioural symptomatology were controlled for. In the same cohort, the authors showed that

BOX 3 Ultra-high risk for psychosis

- The concept of ultra-high risk for psychosis, as a segment of the psychosis continuum coupled with distress and help-seeking, has been studied over the past decade and holds potential for clinicians owing to its high value in providing a window for intervention to prevent psychotic disorders and associated morbidity

- Ultra-high risk individuals are in a prodromal phase of psychosis and they are identified from 'brief limited intermittent psychotic symptoms' (BLIPS) and 'attenuated psychotic symptoms' (APS) bullying predicted psychiatric hospital treatment and use of antipsychotics, but when the analysis was controlled for total psychopathology this effect disappeared for males. The authors identified limitations to their study, including reporting bias, unspecified times of victimisation and a psychiatric diagnosis not determined through a standardised interview. An article comparing the presence of bullying victimisation among patients with a first psychotic episode and a control group (Trotta 2013) showed that patients were approximately twice as likely to report bullying victimisation, and the association remained after controlling for confounders; the study also provides interesting data on the strong relationship between conduct problems and diagnoses of schizophrenia spectrum disorders and affective psychosis, and a link with cannabis use.

\section{Bullying and ultra-high risk}

Individuals at ultra-high risk for psychosis are a useful population in which to study the relationship between trauma and psychosis for two reasons: they allow a follow-up to the emergence of psychotic disorder, and an intrinsic control group (those who do not develop psychosis) at the second time point. In one study, ultra-high risk individuals reported more types of childhood trauma and bullying than the controls, and these experiences were highly correlated with anxiety, depression, negative sense of self and others, and perceived discrimination. Poorer social functioning was more likely to be associated with bullying than with the other types of trauma (Addington 2013). Another study found that total trauma exposure in childhood was positively associated with severity of attenuated psychotic symptoms (in particular grandiosity), and that childhood physical abuse in particular was associated with suspiciousness, suggesting a link with paranoid beliefs (Thompson 2009). In this regard it may be worth focusing in depth on psychotic symptom content to understand the disorder in relation to prior stressful life experiences. The content of humiliating events such as bullying may be linked to the nature of delusions (Catone 2016). Research on the impact of childhood trauma on attenuated psychotic symptoms in 45 individuals (25 males and 20 females) at ultra-high risk for psychosis found a prevalence of $82 \%$ and $42 \%$ for psychological bullying and physical bullying respectively. Furthermore, the study revealed a significant correlation between increased trauma and symptom content such as grandiose thoughts of status and power, feeling watched or followed, negative thoughts regarding the self and hearing non-negative voices (Falukozi 2012). 
Valmaggia et al (2015) confirmed that ultra-high risk people who showed paranoid ideation during a virtual reality experience were more likely to have been bullied in childhood, and Stowkowy et al (2016a) found that ultra-high risk people had experienced more childhood trauma, bullying and perceived discrimination than healthy controls in the North American Prodromal Longitudinal Study 2. The recent development of social media and communications technology has generated another form of peer victimisation - 'cyberbullying' - which seems to have the same negative effects on mental health as traditional bullying. The relationship between cyberbullying and ultra-high risk for psychosis has been investigated in a study involving 50 ultra-high risk young people: $38 \%$ had experienced cyberbullying, and the most frequent types of victimisation reported were bullying via text messages, Facebook and instant messages ('chat') (Magaud 2013).

\section{Bullying and psychotic-like experiences}

Psychotic experiences affect not only people with psychotic disorders: they are also found in the general population. A systematic review by van Os et al (2009) estimated the overall median prevalence of subclinical psychotic symptoms in the general population to be 5.3\% (IQR 1.9-14.4), but the percentage is highest in adolescence (Kelleher 2011). Psychosocial factors such as childhood trauma and stressful life events can act on psychoticlike experiences (PLEs) and predict the onset on psychotic symptoms. Bullying was significantly associated with predisposition to PLEs and these phenomena might increase with negative posttrauma cognitions and an interpersonal context characterised by peer hostility and rejection. The review by van Dam et al (2012) reported a doseresponse relationship with PLEs and frequency, severity and persistence of bullying, and the timing of the stressful experience early in childhood. Adolescents with PLEs suffered a high rate of psychopathological problems (included bullying) during crucial developmental periods. Bullying victimisation at 8 and 10 years of age was a strong predictor of PLEs in a longitudinal study after controlling for prior psychopathology, familial diversity and the child's IQ. Furthermore, the severity and chronicity of the victimisation were the most reliable predictors of transition to psychosis. A subsequent longitudinal study confirmed these findings, showing significant correlations between any role in bullying and psychotic experiences at 18 years of age, with those who were both bullies and victims at greater risk (Wolke 2014). The longitudinal association between bullying and
PLEs was also confirmed in the second and third APMS, which found that bullying was linked with the new onset and the maintenance of persecutory ideation and, to a lesser extent, hallucinations (Catone 2015).

Another longitudinal study looked at different developmental trajectories of psychotic experiences to assess whether bullying and cannabis use may trigger first-onset psychotic symptoms or sustain existing symptoms. A class model was used that allocated participants to subgroups of trajectories on the basis of the elevated/persistent or increasing/ emerging nature of their psychotic experiences. Bullying by peers significantly predicted change in psychotic experiences in adolescents in the 'increase' group, characterised by initially low levels of PLEs that increased over time (Mackie 2013).

Kelleher et al (2013) empirically demonstrated a temporal and bidirectional relationship between childhood trauma and psychosis with the finding that the cessation of childhood stressful life events predicted reduction of PLEs. Horrevorts et al (2014) examined the prevalence of bullying in the classroom (the 'bully climate') and its association with subclinical psychotic symptoms. They found that a higher percentage of classmates involved in bullying weakened the association between victimisation and PLEs: victims may feel social support from other people in the same situation. A recent 35-year longitudinal study confirmed the association between bullying in childhood/ adolescence and psychotic symptoms in adulthood (Boden 2016). Moreover, data showed that children who were bullied also had greater exposure to sexual abuse and other adverse events (socioeconomic disadvantage), poorer cognitive skills, and showed more anxious/withdrawn behaviour and attention problems. Finally, Cristóbal-Narváez et al (2016a) examined individual stress reactivity in the link between bullying and psychosis; they argued that bullying may increase psychotic-like or paranoid reactivity to stress.

\section{Theoretical explanations for the association between bullying and psychosis}

An analysis of the literature allows us to classify explanatory models into three kinds: developmental, biological and cognitive. These models are not wholly distinct or mutually exclusive (Box 4).

\section{Developmental models}

Personal risk factors

There are personal characteristics that increase the likelihood of psychosis that also elevate the possibility of being a victim of bullying. A delay in early development and adjustment difficulties 
BOX 4 Mechanisms of association

Researchers, clinicians and policy makers are interested in how bullying and psychosis may be associated. Distinct trajectories may be involved but, in particular, direct or indirect associations between the two events are both conceivable.

Psychotic symptoms may develop from a traumatic event as a direct reaction to that event, but bullying could lead to the onset of psychotic symptoms through specific affective or cognitive alterations subsequent to previous trauma or through a trajectory that recognises other forms of psychopathology, such as anxiety or depressive disorders (emerging from victimisation).

constitute a premorbid feature of psychotic disorder. Indeed, follow-up studies have shown that motor milestones achieved late in the first 2 years, and poor expressive language ability and social maladjustment at age 7 were predictors of psychotic onset (Isohanni 2000). In addition, factors in the cognitive domain such as poor school performance (repeating a grade, low performance), intellectual impairments such as lower IQ and neuropsychological deficits have been observed among risk factors for psychosis (Bearden 2006). Several lines of evidence support a link between, or a co-presence of, autism spectrum disorder and psychosis (Rapoport 2009). Emotional problems are also involved, and cognitive psychology recognises a central role for internalising problems such as anxiety, depression, social anxiety and mood instability in the subsequent development of psychotic symptoms (Freeman 2003). Bullying victimisation seems to share several of these risk factors, such as pre-existing adjustment difficulties, a diagnosis of autism spectrum disorder (Schroeder 2014), emotional disturbances, and cognitive and intellectual impairment. Victims of bullying also show poor coordination, high levels of internalising problems such as depression and anxiety; they are described as insecure and sensitive, and showing a lack of confidence in social interactions. Children with disabilities, low IQ and intellectual difficulties are more likely to be bullied (Olweus 1993).

\section{Environmental risk factors}

Psychosis and bullying victimisation share a number of environmental factors. A general consensus recognises belonging to a minority, having parents of low socioeconomic status and living in an urban area as risk factors for the development of psychosis (Heinz 2013). Victimisation by bullying is also more frequent among individuals belonging to disadvantaged social classes and settled in urban areas. A study that set out to identify risk factors underlying chronic victimisation emphasised the role of early experience of socioeconomic disadvantage (Bowes 2013). It also found that low maternal warmth, low social support and child maltreatment were associated with victimisation. Parental support is highly correlated with bullying across all kinds of victimisation (direct and indirect). In fact, parents play a central role as they have a dual purpose of ensuring adequate social support for their child and modelling future social behaviour and relationships (Shields 2001). Other non-genetic familial factors implicated in psychosis are being the child of an unwanted pregnancy, maternal stress and depression, paternal psychopathology and disturbances in the parent-child relationship.

Attachment, mentalising, maltreatment and social functioning

The attachment relationship between the child and the caregiver shapes the quality of the child's later social relationships with others. In a pioneering study, Troy \& Sroufe (1987) found links between attachment status at 18 months of age and being bullied by peers at $4-5$ years old; Walden $\&$ Beran (2010) showed that students with low selfperceived quality of attachment to their primary caregivers reported a high frequency of bullying victimisation. Furthermore, attachment quality is involved in resistance/vulnerability to traumatic events. Similar factors and processes appear relevant in the onset of psychosis. In a review of the role of attachment, Gumley et al (2013) concluded that substantial evidence existed to support an association between avoidant attachment and positive and negative symptoms of psychosis, and they found modest evidence of an association between attachment anxiety/preoccupation and positive psychotic symptoms.

Research into social cognition has established that empathy and awareness and regulation of emotions are prerequisites for social skills. Empathy is a broad psychological construct that refers to our ability to feel or consider (think about) the emotional or mental states of others. Therefore, two kinds of empathy, emotional and cognitive, have been studied. Terms such as 'theory of mind' and 'mentalising' include several aspects of emotional and cognitive empathy (Perry 2013). In an elegant study, van Rijn and colleagues (2011) showed that adolescents at ultra-high risk for psychosis had problems in identifying and verbalising their own emotions and appeared socially inadequate, a finding in keeping with an earlier meta-analysis which showed that patients with psychotic symptoms had mentalising impairment (Sprong 2007). Bullying and being 
bullied might also involve misunderstanding of other people's behaviour, and Shakoor et al (2012) found that having poorer theory of mind skills in childhood predicted becoming a victim, bully or both victim and bully in adolescence.

Studies on maltreated children have affirmed that bullied children tend to expect aggression to characterise all relationships and this internalisation influences new interactions, resulting in submissive behaviour to protect them from a potential threat (Shields 2001). Moreover, in middle childhood to early adolescence, emotion regulation can mediate the effect of maltreatment on subsequent victimisation, for example a status of hyperarousal and anxiety that may be adaptive in a violent home is deeply counterproductive in new social settings. These findings suggest that temperament characteristics are involved in determining victimisation status. This is likely to be a dynamic process in which victimisation can modify temperament, thus increasing the risk of further bullying.

Victims tended to be more socially isolated. This is particularly important in adolescence, a key period for new interactions and social demands, when the tendency to withdraw may indicate a difficulty in the development process, framed in an organisational perspective. Functional and social impairment are well known characteristics for individuals who later develop psychosis. Impaired social functioning has been shown as a reliable factor in predicting conversion to psychosis in young people at clinical high risk for psychosis, demonstrating stability over time and independence of clinical state (Cornblatt 2012). Social withdrawal resulting in a noticeable deterioration in social, academic or occupational functioning has always been part of the qualitative descriptions of the states of prodromal schizophrenia, and current advances in research have confirmed this concept, which is one of the classic phenomenological and psychopathological observations of incipient psychosis (Ruhrmann 2010).

\section{Biological models}

The most promising explanations emerge from genetic, neuroendocrine and neurotransmitter studies, together with neuroanatomical findings. Peer victimisation and psychosis proneness may share underlying genetic components. A candidate seems to be a phenotype characterised by enhanced reactivity to emotional stress, as argued by a study in a cohort of twins which found that a disposition to psychosis cosegregates ${ }^{\mathrm{a}}$ with increased reactivity to stress (Lataster 2009). In addition, it has been suggested that the cumulative effect of repeated victimisation leads to a sensitised state characterised by a change in the individual's response to environmental insults (Aiello 2012).

Genetics might also reveal specific genes involved in the onset of trauma-related psychosis in the context of gene-environment $(\mathrm{G} \times \mathrm{E})$ interaction; a possible candidate is the FKBP5 gene already studied in relation to post-traumatic stress disorder. A single nucleotide polymorphism (SNP) in the FK506 binding protein 5 (FKBP5) has been associated with the onset of stress-related mental disorders. FKBP5 is a critical modulator of the feedback loop determining glucocorticoid receptor sensitivity. This gene has been studied in psychotic symptoms. Collip et al (2013) found an interaction between two FKBP5 SNPs and childhood trauma and psychotic symptoms, supporting the role of the FKBP5 genotype in mediating the effects of childhood trauma on mental disorders. In this regard, a recent study by Cristóbal-Narváez et al (2016b) demonstrated that the interplay between the FKBP5 haplotype and bullying was linked with positive psychotic symptoms and this interaction (bullying $\times$ FKBP5) was explained by the stress appraisal of 'being alone because people do not want to be with you'.

The behavioural response to stress is related to functioning of the hypothalamic-pituitary-adrenal (HPA) axis and therefore it has been suggested that bullying could disrupt this system (Vaillancourt 2008). Several researchers have evaluated cortisol levels in bullied children and adolescents and found a blunted salivary cortisol response to stress (Ouellet-Morin 2011). Dysregulation of the HPA axis is also implicated as a vulnerability to psychosis (Aiello 2012).

Increased stress may play many roles in the onset of psychosis. For example, by its effect on dopamine release, heightened emotional reactivity could interfere with the ability to cope with everyday situations. Since release of dopamine increases in response to stress, it would be of interest to investigate further the implication of this relationship in the causation of psychosis, given the link between increased dopamine release and expression of positive psychotic symptoms. Lodge \& Grace (2011) reviewed the evidence in this area and highlighted the role of the ventral hippocampus as a potential site of convergence by which stress may increase symptoms associated with neuropsychiatric disease, including schizophrenia. Indeed, the ventral hippocampus is associated with regulation of both the HPA axis and dopamine neuron activity (Broome 2005).

\section{Cognitive models}

Fowler and colleagues, speculating about the possible pathways between trauma and psychosis, described a. Cosegregation is the tendency for genes in close proximity on the same chromosome to segregate (be inherited) together. 
four trajectories. One of these is the catastrophic interaction hypothesis, which underlines the association between information processing abnormalities and emotional reactions to stress. They write: "problems in contextual processing associated with vulnerability to psychosis may then have the capacity to distort or exaggerate personally significant threat, related anxiety and a range of emotions' (Fowler 2006: p. 116). This explanation highlights two considerations: first, that cognitive models of the relationship between trauma and psychosis are looking with interest at the role of a pre-existing vulnerability to psychosis; second, that an individual's appraisal of trauma creates a context in which positive symptoms of psychosis develop and are maintained.

Two examples of complex cognitive models of positive symptoms of psychosis are found in the study of persecutory delusions and hallucinations.

\section{Persecutory delusions}

Freeman et al (2002) describe a cognitive model of paranoia and persecutory delusions. They explain that persecutory delusions have a common theme of the 'anticipation of danger' and have similar maintaining factors (such as anxiety disorders) that give rise to threat beliefs. In the model, anxiety and depression are assumed to be key emotions in the onset and in the content of delusion. For the authors, the delusion arises as a result of a precipitator such as a life event that causes arousal against a background of vulnerability to psychosis that is expressed by long-term emotional disturbances and sleep problems. The person then feels anomalous experiences (such as perceptual anomalies or thoughts being experienced as voices) that generate an attempt to 'search for meaning'.

The role of cognition on the pathway between childhood trauma and later persecutory delusions is clear from the effect of the emotions triggered by an environmental event such as bullying. Early childhood trauma can contribute to a negative view of the self as being constantly exposed to threat, and to negative schematic beliefs about others; together, these factors have an impact on the vulnerable individual, triggering the onset of psychosis (Garety 2007; Fowler 2012). Therefore, persecutory delusions are formed more easily in someone who has the pre-existing belief that they are a 'soft target' or that the threat was deserved, or who, because of early life experiences, sees other people as hostile. Another explanation involves a particular attributional style that has been found to be associated with the emergence of psychotic symptoms. The locus of control is a cognitive construct that refers to the degree to which individuals have confidence in controlling events that affect them. In a sophisticated analysis it has been shown that bullying has a strong association with psychotic symptoms and the indirect pathway linking the two was represented mainly by having an external locus of control (Fisher 2013).

\section{Hallucinations}

Several cognitive explanations have been proposed to clarify the mechanisms of hallucinations, but there is an overall consensus that these manifestations are the result of an attribution of an event to external causes instead of internal ones. This may be explained by the presence of source monitoring deficits. Source monitoring is a group of metacognitive processes that allow the discrimination of whether events have an internal or external origin. Therefore, a source monitoring deficit would be attributed to a difficulty in discriminating the origin of internal or external events, but also to a misattribution of internal cognitive events to an external source. The research on the relationship between trauma and hallucinations has grown in recent years. It has been postulated that, as in post-traumatic stress disorder, individuals directly re-experience intrusive memories, but in psychosis they misinterpret them (Morrison 2003). Emotional disorders and negative schematic beliefs about the self and others have investigated as mediators between the traumatic experience and the onset of the hallucinations. In a study that tested the association between stressful life events and the onset of hallucinations, the authors stated that "people who have been bullied may subsequently experience anxiety and beliefs about their vulnerability to threat from others. This may trigger voices with threatening harassing contents' (Hardy 2005).

Maladaptive and negative schema, cognitive vulnerability, interpersonal sensitivity and mediation studies

Cognitive models of psychosis have highlighted the role of negative schematic beliefs in the association between psychotic symptoms and adverse events (Fisher 2012). Anilmis et al (2015) analysed the role of negative schematic beliefs about the self and others (as mediators between bullying victimisation and distressing unusual experiences) in 8- to 14-year-olds referred to child and adolescent mental health services. Their findings strongly support the view that negative beliefs following a stressful event may lead to PLEs.

In the wider area of cognitive vulnerability, Jaya et al (2017) demonstrated that negative schemas related to self and others were associated with 
both positive and negative symptoms of psychosis, whereas low perceived social rank and loneliness were associated only with negative symptoms.

Schema therapy is an integrated treatment derived from cognitive, interpersonal, experiential and psychoanalytic therapies that has introduced the concept of core schema, a psychological construct based on memories, emotions and beliefs regarding the self and relationships with others. Stowkowy et al (2016b) demonstrated that maladaptive schemas were most represented in individuals at ultra-high risk for psychosis than in controls, and those who subsequently developed psychosis showed worse maladaptive schemas at the time of transition. Moreover, bullying was positively associated with maladaptive schemas.

Several paths in the association between bullying and psychosis have recently been tested. Bullying has been involved in the relationship between attentiondeficit hyperactivity disorder (ADHD) and psychosis (Hennig 2017). Interpersonal sensitivity (increased awareness of and sensitivity to the emotions and behaviour of others) is a well-recognised mediator in the association between bullying and paranoid ideation. McDonnell et al (2017) demonstrated this association in 64 ultra-high-risk individuals, and Masillo et al (2017) replicated the finding in 147 help-seeking adolescents.

Finally, in an attempt to delineate interactions and directions of causation between multiple mediators in the association between bullying and psychotic symptoms, a recent study that used data from 2000 and 2007 APMS indicated that bullying had a direct effect on worry, mood instability, drug misuse and persecutory delusions. It found that worry and mood instability may have a role as mediators in the indirect effect leading from bullying to psychosis, but this direction of causation cannot be definitely established. Depression, anxiety and sleep problems were not involved as mediators, and bullying had only an indirect association with hallucinations via persecutory ideation and depression (Moffa 2017).

These findings may elucidate psychological mechanisms and help to promote the best strategies for targeting psychotherapeutic interventions.

\section{Discussion}

In the past decade there has been a growing body of work on the relationship between bullying victimisation and psychosis. The evidence that bullying can interact with the onset of psychotic symptoms is enhanced by the consistency of the results to date in population-based non-clinical samples. This finding is reinforced given that the strongest associations are found with greater frequency, severity and persistence of bullying.
Several directions for future research are indicated. Taking a social and descriptive approach, psychosis research should examine the specific content of psychotic symptoms (delusions, hallucinations) to see whether it reflects previous experiences. Studies on bullying and individuals at ultra-high risk for psychosis should consider whether being at risk might affect the perception of being bullied (Mayo 2017). For example, having paranoia may make the person more sensitive to misunderstanding the neutral actions of others as bullying. Cyberbullying should be included as a stressor because it is beginning to demonstrate the potential to trigger psychotic disorders (Krishna 2013).

\section{Neurodevelopmental models}

Research on the role of bullying in psychosis cannot be separated from a careful study of the developmental trajectories involved (Box 5). Bentall (2006) argued that a deeper understanding of the origins of psychosis might be obtained by integrating theoretical concepts and developmental psychology; in fact, the idea that psychosis is the final point of abnormal developmental trajectories cannot be explained solely through an understanding of neurobiology.

The complex framework of developmental psychopathology is used to identify early deviations from normal developmental trajectories that might lead to psychiatric illness. Hence, a developmental approach that focuses on the relationship between normal and atypical processes and how they interact over time embodies an interest in diverse domains of functioning and aims to understand the quality of adaptation across the lifespan (Cicchetti 1995).

\section{B0X 5 Neurodevelopmental models}

- The neurodevelopmental model of schizophrenia suggests that the disease is an end point of several neurodevelopmental abnormalities that started before the onset of the illness; bullying may interact with this neurodevelopmental process.

- The traumagenic neurodevelopmental model posits an integration between social, biological and psychological factors; unlike the stress-diathesis model, it emphasises the fact that the environment affects the brain. The trauma, if prolonged, generates a vulnerability to psychosis and oversensitivity to other stress. Bullying victimisation may be a condition that has the characteristics of this type of trauma.

- The psychosis proneness-persistence-impairment model sets out that environmental stressors can cause developmental manifestations of psychosis to become persistent, thus determining clinical impairment 
The risk factors shared between bullying victimisation and psychosis are numerous. In principle, assessment of the relationship between risk factors and psychopathology offers the possibility of understanding fundamental developmental processes. However, the presence of multiple risk factors in this case makes it difficult to disentangle the links between them and also the relative effects of moderators and outcomes (Cicchetti 1995). Nevertheless, it is clear that people at greater risk of developing psychosis also are likely to have an enhanced risk of being victims of bullying in their life. In fact, a profile characterised by poor early adjustment, cognitive and emotional disturbances, the presence of autism spectrum disorder or difficulties in the social domain, and environmental factors such as sociodemographic status or even a traumatic or neglectful environment is shared between the two conditions. The current evidence enables a more complex debate to be raised: is bullying a social trigger of psychosis, able to divert the individual's developmental pathway towards this mental illness, or is it a direct manifestation of the inherent vulnerability for psychosis along this pathway? In this regard, Shakoor and colleagues (2014), in a large prospective community-based twin study, found that bullying and paranoia are almost wholly linked via a genetic pattern and that individuals prone to bullying are also phenotypically predisposed to paranoia. Cunningham et al (2016) hypothesised that the development of specific psychotic symptoms in adults who were bullied in childhood may be explained better by the cumulative effect of adversities and emotional and behavioural problems in that period rather than as a causal and direct effect of bullying.

Although bullying victimisation is linked to psychosis, the association is not specific, given that stressors such as victimisation are linked to a wide range of mental disorders. The developmental perspective offers a theoretical construct that provides support to this statement; it refers to the concepts of 'equifinality' - different conditions can lead to the same outcome - and 'multifinality' - the same condition can lead to many different outcomes. The study of the risk trajectories involved in the association may explain whether there is any specificity of the pathways involved.

\section{Integrating cognitive and biological models}

Any synthesis of the literature leads to the question of how cognitive and biological models could be integrated. Biological causes offer explanations in terms of psychopathology, how these alterations are transformed into psychological difficulties, and how the environment along with biological alterations can contribute to these. In the biological models section of this article, we focused on the role attributed to the limbic structures, noting that abnormal input from these results in increased salience and increased emotional reactivity (salience is the relevance that is attributed to an event, and increased salience can lead to psychotic symptoms). Bullying may have the potential to cause subthreshold dysregulation in these systems (Arseneault 2010), leading to an excessive appraisal of the victimisation. This in turn increases the stress and dysregulation, so that the individual attributes further aberrant salience to the bullying and creates meaningful connections that are clearly psychotic. Alongside this exposure, a negative view of the self, others and the world develops which may explain the onset of psychotic symptoms.

\section{Clinical implications}

Several implications arise from this discussion. First, bullying and other stressful events, such as interpersonal trauma and physical and sexual abuse, should be included in the mental health assessment, focusing on such experiences in childhood and adolescence. Second, the association between bullying and psychosis has preventive and therapeutic consequences. Prevention of bullying, perhaps through educational and support programmes in schools, has the potential to reduce the experience of psychotic symptoms. Third, psychotherapy can help individuals to cope with the traumatic consequences of bullying and, at a more inner level, can aim to change negative cognitive schemas and modify the trajectory of early psychopathology, as well as reducing the potency of bullying to produce psychotic symptoms. Cognitive-behavioural therapy (CBT) in particular can help individuals with psychosis to make sense of their experiences by linking emotions, beliefs and traumatic events with psychotic symptoms. It can also allow them to find alternative explanations of their experiences (Callcott 2006).

\section{Acknowledgement}

We thank the staff of Warneford Library, Oxford, for their help in retrieving books and journals.

\section{References}

Addington J, Stowkowy J, Cadenhead KS, et al (2013) Early traumatic experiences in those at clinical high risk for psychosis. Early Intervention in Psychiatry, 7: 300-5.

Aiello G, Horowitz M, Hepgul N, et al (2012) Stress abnormalities in individuals at risk for psychosis: a review of studies in subjects with familial risk or with 'at risk' mental state. Psychoneuroendocrinology, 37: 1600-13.

Anilmis JV, Stewart CS, Roddy S, et al (2015) Understanding the relationship between schematic beliefs, bullying, and unusual experiences in 8-14 year olds. European Psychiatry, 30: 920-3. 
Arseneault L, Bowes L, Shakoor S (2010) Bullying victimization in youths and mental health problems: 'much ado about nothing'? Psychological Medicine, 40: 717-29.

Bearden CE, Mayer SE, Loewy RL, et al (2006) The neurodevelopmental model of schizophrenia: updated. In Developmental Psychopathology Volume 3: Risk, Disorder, and Adaptation (2nd edn) (eds D Cicchetti, DJ Cohen): 542-69. John Wiley \& Sons.

Bebbington PE, Bhugra D, Brugha T, et al (2004) Psychosis, victimisation and childhood disadvantage: evidence from the second British National Survey of Psychiatric Morbidity. British Journal of Psychiatry, 185: 220-6.

Bentall RP (2006) The environment and psychosis: rethinking the evidence. In Trauma and Psychosis: New Directions for Theory and Therapy (eds W Larkin, P Morrison): 7-23. Routledge.

Boden JM, Van Stockum S, Horwood LJ et al (2016) Bullying victimization in adolescence and psychotic symptomatology in adulthood: evidence from a 35-year study. Psychological Medicine, 46: 1311-20.

Bowes L, Maughan B, Ball H, et al (2013) Chronic bullying victimization across school transitions: the role of genetic and environmental influences. Developmental Psychopathology, 25: 333-46

Broome MR, Woolley JB, Tabraham P, et al (2005) What causes the onset of psychosis? Schizophrenia Research, 79: 23-34.

Callcott P, Turkington D (2006) CBT for traumatic psychosis. In Trauma and Psychosis: New Directions for Theory and Therapy (eds W Larkin, P Morrison): 222-38. Routledge.

Catone G, Marwaha S, Kuipers E, et al (2015) Bullying victimisation and risk of psychotic phenomena: analyses of British national survey data. Lancet Psychiatry, 2: 618-24.

Catone G, Pisano S, Broome M, et al (2016) Continuity between stressful experiences and delusion content in adolescent with psychotic disorder: a pilot study. Scandinavian Journal of Child and Adolescent Psychiatry and Psychology, 4: 14-22.

Cicchetti D, Cohen DJ (eds) (1995) Developmental Psychopathology. Vol 1: Theory and Method. John Wiley \& Sons.

Collip D, Myin-Germeys I, Wichers M, et al (2013) FKBP5 as a possible moderator of the psychosis-inducing effects of childhood trauma. British Journal of Psychiatry, 202: 261-8.

Cornblatt BA, Carrion RE, Addington J, et al (2012) Risk factors for psychosis: impaired social and role functioning. Schizophrenia Bulletin, 38: 1247-57.

Cristóbal-Narváez P, Sheinbaum T, Ballespi S, et al (2016a) Impact of adverse childhood experiences on psychotic-like symptoms and stress reactivity in daily life in nonclinical young adults. PloS One, 11(4): e0153557.

Cristóbal-Narváez P, Sheinbaum T, Rosa A, et al (2016b) The interaction between childhood bullying and the FKBP5 gene on psychotic-like experiences and stress reactivity in real life. PLoS One, 11(7): e0158809.

Cunningham T, Hoy K, Shannon C (2016) Does childhood bullying lead to the development of psychotic symptoms? A meta-analysis and review of prospective studies. Psychosis, 8: 48-59.

Falukozi E, Addington J (2012) Impact of trauma on attenuated psychotic symptoms. Psychosis, 4: 203-12.

Fisher HL, Appiah-Kusi E, Grant C (2012) Anxiety and negative selfschemas mediate the association between childhood maltreatment and paranoia. Psychiatry Research, 196: 323-4.

Fisher HL, Schreier A, Zammit S, et al (2013) Pathways between childhood victimization and psychosis-like symptoms in the ALSPAC birth cohort. Schizophrenia Bulletin, 39: 1045-55.

Fowler D, Freeman D, Steel C et al (2006) The catastrophic interaction hypothesis: how do stress, trauma, emotion and information processing abnormalities lead to psychosis? In Trauma and Psychosis: New Directions for Theory and Therapy (eds W Larkin W, P Morrison): 101-24. Routledge.

Fowler D, Hodgekins J, Garety P, et al (2012) Negative cognition, depressed mood, and paranoia: a longitudinal pathway analysis using structural equation modeling. Schizophrenia Bulletin, 38: 1063-73.

Freeman D, Garety PA, Kuipers E, et al (2002) A cognitive model of persecutory delusions. British Journal of Clinical Psychology, 41: 331-47.
Freeman D, Garety PA (2003) Connecting neurosis and psychosis: the direct influence of emotion on delusions and hallucinations. Behaviour Research and Therapy, 41: 923-47.

Fusar-Poli P, Bonoldi I, Yung AR, et al (2012) Predicting psychosis: meta-analysis of transition outcomes in individuals at high clinical risk. Archives of General Psychiatry, 69: 220-9.

Garety PA, Bebbington P, Fowler D, et al (2007) Implications for neurobiological research of cognitive models of psychosis: a theoretical paper. Psychological Medicine, 37: 1377-91

Gumley Al, Taylor HE, Schwannauer M, et al (2013) A systematic review of attachment and psychosis: measurement, construct validity and outcomes. Acta Psychiatrica Scandinavica, 129: 257-74.

Hardy A, Fowler D, Freeman D, et al (2005) Trauma and hallucinatory experience in psychosis. Journal of Nervous and Mental Disease, 193: 501-7. Heinz A, Deserno L, Reininghaus U (2013) Urbanicity, social adversity and psychosis. World Psychiatry, 12: 187-97.

Hennig T, Jaya ES, Lincoln TM (2016) Bullying mediates between attention-deficit/hyperactivity disorder in childhood and psychotic experiences in early adolescence. Schizophrenia Bulletin, 43: 1036-44.

Horrevorts EM, Monshouwer K, Wigman JT, et al (2014) The relation between bullying and subclinical psychotic experiences and the influence of the bully climate of school classes. European Child \& Adolescent Psychiatry, 23: 765-72

Isohanni M, Jones P, Kemppainen L, et al (2000) Childhood and adolescent predictors of schizophrenia in the Northern Finland 1966 birth cohort: a descriptive life-span model. European Archives of Psychiatry and Clinical Neuroscience, 250: 311-9.

Jaya ES, Ascone L, Lincoln TM (2016) Social adversity and psychosis: the mediating role of cognitive vulnerability. Schizophrenia Bulletin, 43: $557-65$

Kelleher I, Cannon M (2011) Psychotic-like experiences in the general population: characterizing a high-risk group for psychosis. Psychological Medicine 41: 1-6.

Kelleher I, Keeley H, Corcoran P, et al (2013) Childhood trauma and psychosis in a prospective cohort study: cause, effect, and directionality. American Journal of Psychiatry, 170: 734-41.

Krishna N, Fischer BA, Miller M, et al (2013) The role of social media networks in psychotic disorders: a case report. General Hospital Psychiatry, 35: 576.e1-e2.

Lataster T, Wichers M, Jacobs N, et al (2009) Does reactivity to stress cosegregate with subclinical psychosis? A general population twin study. Acta Psychiatrica Scandinavica, 119: 45-53

Lodge DJ, Grace AA (2011) Developmental pathology, dopamine, stress and schizophrenia. International Journal of Developmental Neuroscience, 29: 207-13.

Mackie CJ, O'Leary-Barrett M, Al-Khudhairy N, et al (2013) Adolescent bullying, cannabis use and emerging psychotic experiences: a longitudinal general population study. Psychological Medicine, 43: 1033-44.

Magaud E, Nyman K, Addington J (2013) Cyberbullying in those at clinical high risk for psychosis. Early Intervention in Psychiatry, 7: 427-30.

Masillo A, Valmaggia LR, Saba R, et al (2017) Interpersonal sensitivity, bullying victimization and paranoid ideation among help-seeking adolescents and young adults. Early Intervention in Psychiatry, 30 May. doi: 10.1111/eip.12447 [Epub ahead of print]

Mayo D, Corey S, Kelly LH, et al (2017) The role of trauma and stressful life events among individuals at clinical high risk for psychosis: a review. Frontiers in Psychiatry, 8: 55.

McDonnell J, Stahl D, Day F, et al (2017) Interpersonal sensitivity in those at clinical high risk for psychosis mediates the association between childhood bullying victimisation and paranoid ideation: a virtual reality study. Schizophrenia Research, 26 April. doi: 10.1016/j. schres.2017.04.029 [Epub ahead of print]

Moffa G, Catone G, Kuipers J, et al (2017) Using directed acyclic graphs in epidemiological research in psychosis: an analysis of the role of bullying in psychosis. Schizophrenia Bulletin, 17 May. doi: 10.1093/ schbul/sbx013 [Epub ahead of print]

\section{MCO answers \\ 1 a 2 a 3 c $\quad 4$ c 5 e}


Morrison AP, Petersen T (2003) Trauma, metacognition and predisposition to hallucinations in non-patients. Behavioural and Cognitive Psychotherapy, 31: 235-46.

Olweus D (1993) Bullying at School: What We Know and What We Can Do. Blackwell.

Ouellet-Morin I, Odgers CL, Danese A, et al (2011) Blunted cortisol responses to stress signal social and behavioral problems among maltreated/bullied 12-year-old children. Biological Psychiatry, 70: 1016-23.

Perry A, Shamay-Tsoory SG (2013) Understanding emotional and cognitive empathy: a neuropsychological perspective. In Understanding Other Minds: Perspectives from Developmental Social Neuroscience (eds S Baron-Cohen, M Lombardo, H Tager-Flusberg): 178-94. OUP.

Rapoport J, Chavez A, Greenstein D, et al (2009) Autism spectrum disorders and childhood-onset schizophrenia: clinical and biological contributions to a relation revisited. Journal of the American Academy of Child \& Adolescent Psychiatry, 48: 10-8.

Reeder FD, Husain N, Rhouma A, et al (2017) The relationship between childhood trauma and adult psychosis in a UK Early Intervention Service: results of a retrospective case note study. Neuropsychiatric Disease and Treatment, 13: 269-73.

Ruhrmann S, Schultze-Lutter F, Salokangas RK, et al (2010) Prediction of psychosis in adolescents and young adults at high risk: results from the prospective European prediction of psychosis study. Archives of General Psychiatry, 67: 241-51.

Schroeder JH, Cappadocia MC, Bebko JM, et al (2014) Shedding light on a pervasive problem: a review of research on bullying experiences among children with autism spectrum disorders. Journal of Autism and Developmental Disorders, 44: 1520-34

Shakoor S, Jaffee SR, Bowes L, et al (2012) A prospective longitudinal study of children's theory of mind and adolescent involvement in bullying. Journal of Child Psychology and Psychiatry, 53: 254-61.

Shakoor S, McGuire P, Cardno AG, et al (2014) A shared genetic propensity underlies experiences of bullying victimization in late childhood and self-rated paranoid thinking in adolescence. Schizophrenia Bulletin, 41: 754-63.

Shields A, Cicchetti D (2001) Parental maltreatment and emotion dysregulation as risk factors for bullying and victimization in middle childhood. Journal of Clinical Child Psychology, 30: 349-63.

Sprong M, Schothorst P, Vos E, et al (2007) Theory of mind in schizophrenia: meta-analysis. British Journal of Psychiatry, 191: 5-13.

Stowkowy J, Liu L, Cadenhead KS, et al (2016a) Early traumatic experiences, perceived discrimination and conversion to psychosis in those at clinical high risk for psychosis. Social Psychiatry and Psychiatric Epidemiology, 51: 497-503.

Stowkowy J, Liu L, Cadenhead KS, et al (2016b) Core schemas in youth at clinical high risk for psychosis. Behavioural and Cognitive Psychotherapy, 44: 203-13.

Thompson JL, Kelly M, Kimhy D, et al (2009) Childhood trauma and prodromal symptoms among individuals at clinical high risk for psychosis. Schizophrenia Research, 108: 176-81.

Trotta A, Di Forti M, Mondelli V, et al (2013) Prevalence of bullying victimisation amongst first-episode psychosis patients and unaffected controls. Schizophrenia Research, 150: 169-75.

Trotta A, Murray RM, Fisher HL (2015) The impact of childhood adversity on the persistence of psychotic symptoms: a systematic review and metaanalysis. Psychological Medicine, 45: 2481-98.

Troy M, Sroufe LA (1987) Victimization Among preschoolers: role of attachment relationship history. Journal of the American Academy of Child \& Adolescent Psychiatry, 26: 166-72.

Vaillancourt T, Duku E, Decatanzaro D, et al (2008) Variation in hypothalamic-pituitary-adrenal axis activity among bullied and nonbullied children. Aggressive Behavior, 34: 294-305

Valmaggia LR, Day FL, Kroll J, et al (2015) Bullying victimisation and paranoid ideation in people at ultra high risk for psychosis. Schizophrenia Research, 168: 68-73.

van Dam DS, van der Ven E, Velthorst E, et al (2012) Childhood bullying and the association with psychosis in non-clinical and clinical samples: a review and meta-analysis. Psychological Medicine, 42: 2463-74.

van Os J, Linscott RJ, Myin-Germeys I, et al (2009) A systematic review and meta-analysis of the psychosis continuum: evidence for a psychosis proneness-persistence-impairment model of psychotic disorder. Psychological Medicine, 39: 179-95.

van Rijn S, Schothorst P, Wout M,et al (2011) Affective dysfunctions in adolescents at risk for psychosis: emotion awareness and social functioning. Psychiatry Research, 187: 100-5.

Varese F, Smeets F, Drukker M,et al (2012) Childhood adversities increase the risk of psychosis: a meta-analysis of patient-control, prospective- and cross-sectional cohort studies. Schizophrenia Bulletin, 38: 661-71.

Walden LM, Beran TN (2010) Attachment quality and bullying behavior in school-aged youth. Canadian Journal of School Psychology, 25: 5-8.

Wolke D, Lereya ST, Fisher HL, et al (2014) Bullying in elementary school and psychotic experiences at 18 years: a longitudinal, population-based cohort study. Psychological Medicine, 44: 2199-211.

\section{$\mathrm{MCOs}$}

Select the single best option for each question stem

\section{The prevalence of psychotic-like} experiences (PLEs) in the general population is:

a $5 \%$, but in adolescence is higher

b $7 \%$, but in adolescence is lower

c $4 \%$, but in adolescence is higher

d $4 \%$, but in adolescence is lower

e $5 \%$ in adulthood and adolescence.

\section{Bullying is:}

a an aggressive behaviour that happens in a context of imbalance of powe

b a normal developmental event

c a dispute between two children of equal strength

$\mathrm{d}$ an isolated event

e none of the above.
3 Bullying by peers significantly predicts changes in psychotic experiences in:

a children who have separation anxiety

$\mathrm{b}$ adolescents with intellectual disability

c adolescents with increasing/emerging psychotic experiences and substance misuse

d children of divorced parents

e adolescents who self-harm.

4 The principal purported biological mechanism involved in the association between bullying and psychosis is:

a serotonin decrease

b glutamate dysregulation

c HPA axis dysregulation

$\mathrm{d}$ dopamine reduction

e none of the above.
5 Cognitive models of psychosis recognise:

a the exclusive role of genetic factors

$\mathrm{b}$ the exclusive role of psychological factors

c the central role of the affects

$\mathrm{d}$ an interplay between genetic and psychological factors.

e $c$ and $d$ 